\title{
Freeze-Float System for High-throughput Measurement of Ice
}

\section{Nucleation}

Authors: Yuki Kamijo, ${ }^{1}$ Ratmir Derda ${ }^{2 *}$

1. Department of Chemistry, The University of Tokyo, Bunkyo, Tokyo 113-0033, Japan

2. Department of Chemistry, University of Alberta, Edmonton, AB T6G 2G2, Canada

* Corresponding author

\begin{abstract}
In this publication, we developed the high throughput screening implementation of freeze-float selection platform system we established in the previous publication. The goal of this publication is to expand the system to higher throughput and accuracy. In the following sections, we describe the steps for automated droplets generations, adaptation of freeze-float selection using controlled and uniform temperature cooling system, and semi-automated droplets detection program. We aimed to improve previously published system to add functional advantages, such as; a) increased efficiency of the screening with fewer manipulation steps; b) increased accuracy of measurement due to the increased sample size; c) increased uniformity of temperature distribution by incorporating the controlled-rate freezer. In the following sections, we describe the steps for automated droplets generations, freeze-float system adaptations using controlled and uniform temperature cooling system, and semi-automated droplets detection program.
\end{abstract}




\section{Introduction}

In the previous publication, we reported a first proof-of-concept implementation of freezefloat selection system, ${ }^{1}$ which was able to identify the ice-nucleation event in droplets that contained ice-nucleating entities. $1 \mathrm{~cm}^{2}$ geometry cuvette system successfully accommodated up to $50 \times 1 \mu \mathrm{L}$ droplets at once and separated the samples that have ice-nucleating activity from those do not. However, due to the low thermal conductivity of the silicone oil, it was not possible to increase the footprint of the container and accommodate a larger number of droplets without introducing a significant thermal lag. The throughput could be increased by setting up multiple cuvettes in parallel, but setting up more than 5-10 cuvettes in the same freezer system was difficult, and again, resulted in non-uniform results; for example, in setting up 10 cuvettes at the same time, we observed a difference of a few degrees' between cuvettes. To improve the uniformity of the temperature among multiple cuvettes, the system requires the good heat conductor between cuvettes.

High throughput screening (HTS) in multi-well plates miniaturization and automation of biochemical assays is well-established method to enable a large number of parallel measurements. HTS has been developed originally for drug discovery in the laboratory or pharmaceutical industry to screen the increasing number of potential targets and therapeutic compound libraries. ${ }^{2-5}$ The transition from the low-throughput assay to the HTS results in highly efficient and integrated system, which includes the shortened screening time, reduced amount of sample and reagent, and lower costs of the overall process. Miniaturization using 3456-well microtiter plate made it possible to carry out more than 100,000 assays per day and volume of less than $5 \mu$ L.6, 7 Combination of the automated liquid dispensing and plate handling robotics enabled even higher throughput and reproducibility while the increasing number of assays. ${ }^{8,9}$ To process the large data 
sets associated with the HTS, robust signal detection and statistical analysis systems have also been developed. ${ }^{10}$

Droplets-based assays represent another form of high-throughput assay. It offers the advantage of chemical and physical compartmentalization to avoid cross-contamination of the samples by segmenting the uniform droplets by the immiscible carrier fluid. Successful examples of the droplet microfluidic assays such as crystallization study ${ }^{11}$ or single cell screening of enzyme production $^{12,13}$ reported a million fold reduction of reagent consumption and 300 -folds increase in throughput when compared to the HTS-multiwell assays. Droplet-based assays have also been employed to measure tens of thousands ice nucleation events at one experiment. ${ }^{12,14}$ Microfluidic droplet based cell and virion counting techniques are also reported. ${ }^{15}$ Droplet based analysis became a commercial success in digital polymerase chain reaction (dPCR) ${ }^{16}$ and single-cell genomics. ${ }^{17,18}$ This technology successfully increased the sensitivity of the absolute nucleic acid quantification by compartmentalizing the individual DNA molecule into uniform droplets. When compared to HTS plate-based assays, the drawback of droplet systems is difficult in measuring a large number of droplets of arbitrary compositions.

Preparation of polydisperse silicone oil emulsions by simple mixing have been used in biochemical applications. ${ }^{19}$ Despite the ease of manufacturing, polydispersity is detrimental in icenucleation studies because the freezing temperature is directly proportional to the logarithm of droplets diameter. ${ }^{20}$ The polydispersity of the droplets can directly influence the accuracy of the freezing measurement. Swanson and co-worker observed that the droplet diameter and homogeneous freezing temperature can be expressed as

$$
y=\ln (x)-26.8
$$


where $y$ is nucleation temperature and $x$ is droplet radius. ${ }^{21}$ This approximation will lead to $2.3{ }^{\circ} \mathrm{C}$ difference of mean freezing temperature between the $1 \mathrm{~mm}$ diameter and $0.1 \mathrm{~mm}$ diameter droplets. Microfluidics can produce uniform droplets but interfacing of microfluidics and microtiter well plates used in HTS is not obvious and not trivial. Based on this knowledge, we produced uniform size droplets via the commercially available automated pipetting system and confirmed the monodispersity by image analysis.

To obtain the reliable freezing temperature, sample number is also important. Confidence interval of the freezing temperature of droplets was calculated via the formula:

$$
n \approx(2 k / C I)^{2} s^{2}
$$

$n$ is the sample number, $k$ is the value for desired confidence level (1.96 for 95\% confidence), $C I$ is the confidence interval, and $s$ is the standard deviation of the outcome of interest. From the obtained value, we concluded that more than 30 droplets sample is sufficient to observe the reliable freezing temperature.

We introduced the robotic liquid transfer system Biomek® 3000 (Beckman Coulter, Indianapolis, IN) to enable the automated parallel generation of droplets in wells of a multi-well plate. Biomek 3000 replaced manual generation of droplets by repeat pipetting employed in our previous report. This robotic system provides liquid handling operations with a spatial positioning ability compatible with many multi-well plate geometries. Simple modification to the Biomek program allowed changing the parameters of droplets generation including droplets number or droplets size.

From the prior study, the droplet freezing is hypothesized to be a random nucleation process which can be accelerated in the presence of ice nucleators. Due to the stochastic freezing behavior, the freezing spectra in droplet freezing assays usually shows inherent distributions, 
which results in a spread of the freezing temperature among droplets even when all the droplets have identical size and contain the same number of the INA species. ${ }^{22}$ This dispersion makes it difficult to determine the ice nucleating temperature from a small number of samples. By expanding the size of the assay vessel from a $1 \mathrm{~cm}^{2}$ footprint cuvette that contains up to 50 droplets to the 48 cryovials that can contain up to 40 droplets ( 40 droplets $\times 48$ vials $=1920$ droplets $)$, the throughput can be increased by the factor of 38 . The cooling setup employed the metal cooling plate and sample holder to achieve the homogeneous cooling through the vial to vial.

Wills and co-workers have reported that the droplet freezing assays with cooling rates from 0.8 to $10 \mathrm{~K} / \mathrm{min}$ produced nucleation rates fit in a single line, ${ }^{23}$ which suggested that the difference in cooling rate had minimum effect on the nucleation rate coefficients. However linear cooling rate is important for the droplet freezing assay in the stochastic model of heterogeneous nucleation

measurement. ${ }^{23}$ Based on this report, we conducted the freeze-float experiment incorporating a constant rate freezer Asymptote VIA freezer. This commercially available instrument affords a constant rate cooling with high precision down to $-0.5^{\circ} \mathrm{C} / \mathrm{min}$. We equipped this bench-top instrument with custom-made glass lid to enable automated image recording by generic digital camera.

\section{Results}

\section{Automated droplets generation}

To implement the freeze-float selection at the higher throughput scale, we first tested the 96 deep well plate which is compatible with the robotic liquid handling machine Biomek 3000 (Figure 1 A-B). We used the same silicone oil buoyant layer with $1 \%$ silicone surfactant system and HFE-7500 cushion layer as we used in the previous publication. 
We dispensed $30 \times 1 \mu \mathrm{L}$ droplets into the silicone oil layer of 96 deep well plate using Biomek 3000. At a standard dispensing rate 1 droplet per 2 seconds, a pipetting arm successfully generated 40 uniform droplets in 96 wells (up to $~ 3800$ droplets at once) in $\sim 15$ minutes. The rate can be further accelerated by using a multi-head dispenser.

Uniform volume is the key for quantitative detection of ice-nucleation events. To assess the droplets dispersity, we prepared the $601 \mu \mathrm{L}$ droplets with fluorescein dye by the Biomek 3000 in the silicone oil in the 12 well plate filled with silicone oil and acquired the fluorescent image by ChemiDoc MP (Bio-Rad, CA) (Figure 1C). The droplet diameters were measured by using Matlab automated circle detection program Droplet_Dispersion.m (Source Code S1). This program detects the circles in image in an automated manner and analyze the diameter of each droplets. The polydispersity of the 60 droplets defined as standard deviation of the radii of the droplets (here $2.86 \times 10^{-5}$ ) divided by the mean radius (here $3.10 \times 10^{-4} \mathrm{~m}$ ) was $9.2 \%$, which indicated that the droplets generated by the robotic system have sufficiently uniform volume (Figure 1 D-E). Swanson and co-worker observed that the droplet diameter and homogeneous freezing temperature can be expressed as

$$
y=\ln (x)-26.8
$$

where $y$ is nucleation temperature and $x$ is droplet radius. ${ }^{21}$ Based on the standard deviation of the radii of the droplets, we expected no more than $\pm 0.09{ }^{\circ} \mathrm{C}$ dispersion in the measurement of the nucleation temperature.

We took several precautions to eliminate droplets coalescence. To avoid contact with the plastic wall which can cause the droplets destabilization, the HFE-7500 was always placed at the bottom of the system as a cushion layer. The static electricity of the Biomek 3000 which could cause coalescence of the droplets was removed by using Auto-set Ion Pump (\#19500, Charles 
Water, MA). This machine is bench-top equipment that discharges static potential accumulation caused by the Biomek 3000 operation. Also when we move the droplet system, wet filter paper was placed below the plastic plate to minimize the accumulation of static electricity.

We conducted freeze-float validation experiment of the system implementing Biomek 3000. The Biomek 3000 successfully prepared 30 aqueous droplets with $1 \mathrm{mg} / \mathrm{mL}$ Snomax colored with yellow fluorescein dye in a 96 deep well system. We placed this system in the commodity freezer employed in the previous publication, and recorded the droplets freezing and floating behaviour (Figure 2). The INA droplets showed droplet freezing behaviour with a good agreement with the previous report.

We used Python script KStest.ipynb (Source Code S3) to compare the freezing temperature distribution of droplets from two different samples. Briefly, this script compares cumulative distributions of the freezing temperature from two samples and calculate the p-value using Kolmogorov-Smirnov test to show if the freezing temperature profile of the two samples are drawn from the same distributions.

To achieve the high throughput screening, it is necessary to establish the multi-well platform that can give uniform cooling through all wells in a plate. However, due to the low thermal conductivity of the silicone oil and geometry of the 96 deep well plate, it was difficult to avoid the cell to cell thermal gradient. The freezing temperature in cells in the same deep well plate showed p-value of $4.4 \times 10^{-7}$ at constant temperature $-28^{\circ} \mathrm{C}$ generic freezer (Figure 3 ). The thermal gradient was decreased $(\mathrm{p}=0.31)$ with linear cooling $\left(-0.25^{\circ} \mathrm{C} / \mathrm{min}\right)$ compared to the constant temperature cooling, however it significantly increased the range of freezing time, and mean freezing time between the cells were different (47.4 and $50.6 \mathrm{~min}$ ). We concluded that using these 96 deep well plate approaches as the screening platform is not a promising path. 


\section{Asymptote VIA freezer}

To achieve the constant rate cooling of the freeze-float system and better uniformity of the cell to cell temperature, we employed VIA Freeze ${ }^{\mathrm{TM}}$ Research controlled rate freezer (Asymptote, Cambridge, UK). The freezer successfully provided high accuracy control of the cooling temperature and accommodate up to $48 \times 1 \mathrm{~mL}$ cryovials in an instrument-specific metal holder. Each cryovial contained $50 \mu \mathrm{L}$ of HFE-7500 at the bottom of the cryovial as a cushion layer, $1 \mathrm{~mL}$ silicone oil with $1 \%$ surfactant floating layer, and 20 aqueous droplets with $1 \mathrm{mg} / \mathrm{mL}$ Snomax marked with fluorescein, and 20 droplets of INA free milli Q water marked with Coomassie Brilliant Blue dye. Similar to the previous observations, the droplets with INA froze at -7.1 $\pm 0.59{ }^{\circ} \mathrm{C}$ and droplets without INA did not freeze until the temperature reached to $-12.3^{\circ} \mathrm{C}$ in the Asymptote freezer (Figure 4). This observation suggested that the controlled cooling rate provided better detection of INA compared to the freeze-float selection in a commodity freezer system (mean temperature $-7.5 \pm 0.97^{\circ} \mathrm{C}$ ).

To evaluate the uniformity of the cooling between cells at different positions, we compared the temperature at which $1 \mathrm{~g} / \mathrm{mL}$ Snomax droplets froze in Asymptote controlled rate freezer system and 96 deep well in generic freezer. We used python script KStest.ipynb (Source Code S3) to compare the freezing temperature distributions. We observed well to well variability and interwell dispersion in the freezing time in the 96 deep well system $(\mathrm{p}=0.31$, standard deviation 1.6), whereas the distribution of freezing of droplets in Asymptote freezer (Figure 5) showed less difference of well to well freezing temperature $(\mathrm{p}=0.83$, standard deviation 0.73$)$ and overall $36 \%$ lower dispersion (Figure 6). 


\section{Conclusion}

To increase the efficiency and accuracy of the freeze-float system from the previous publication, we employed the automated droplets dispensing system and controlled rate freezer. By using Biomek 3000 liquid handling system, theoretically generates up to $~ 3800$ uniform $1 \mu \mathrm{L}$ droplets in the 96 well plate freeze-float system. The droplets generated by this system and contained INA showed similar freezing and floating behaviour to the experiment we conducted in the previous publication, which indicates that this system can be implemented to the high throughput freeze-float system. However, when the system needs to be transferred certain distance, for example from one laboratory to another, the droplet stability was not sufficient and static electricity caused by frictions caused coalescing. Due to this problem, it was not successful to combine the droplets generation system.

Next, we implemented the controlled-rate Asymptote freezer system to enlarge the capacity of the droplets and improve the accuracy of the system. The cell to cell thermal delay was improved by the constant linear cooling (from $\mathrm{p}=4.4 \times 10^{-7}$ to $\mathrm{p}=0.31$ ). To confirm the accuracy of the screening system, the detection threshold of the INA concentration was investigated. At the threshold concentration, controlled-rate cooling system showed better differentiation accuracies ( $\mathrm{p}$ $\left.=4.4 \times 10^{-7}\right)$ compared to the commodity freezer $(\mathrm{p}=0.83)$.

The throughput of the described droplet-based screening enables 50 different samples, measuring 40 droplets each. When combined with automated liquid handling, in principle, this system is suitable for medium throughput, high confidence measurement of 100-1000 unique chemical compositions or concentrations. While the unbiased discovery of INAs from a large chemical library remains a challenge in this setup alone, it can serve as a robust validation step for novel putative INA compositions emanating from large-scale genetically-encoded screens. 


\section{Acknowledgements}

We thank Dr. Jason Acker for the assistance with the Asymptote VIA freezer studies. We thank Marlin Van Zandt, the Manager of Edmonton Snow Valley Ski Club for the sample of Snomax. This work was supported by research grants from the Natural Science and Engineering Research Council of Canada (NSERC \#492943).

\section{Notes and References}

$\dagger$ Electronic Supplementary Information (ESI) available: protocols, Supplementary Figures S1S11 and Source Code S1-S3.

1. Kamijo, Y.; Derda, R., Freeze-Float Selection of Ice Nucleators. Langmuir 2019, 35 (2), 359-364.

2. Pereira, D. A.; Williams, J. A., Origin and evolution of high throughput screening. British Journal of Pharmacology 2007, 152 (1), 53-61.

3. Collins, K. D.; Gensch, T.; Glorius, F., Contemporary screening approaches to reaction discovery and development. Nature Chemistry 2014, 6 (10), 859-871.

4. Hughes, J. P.; Rees, S.; Kalindjian, S. B.; Philpott, K. L., Principles of early drug discovery. British Journal of Pharmacology 2011, 162 (6), 1239-1249.

5. Kansy, M.; Senner, F.; Gubernator, K., Physicochemical High Throughput Screening:

Parallel Artificial Membrane Permeation Assay in the Description of Passive Absorption Processes. Journal of Medicinal Chemistry 1998, 41 (7), 1007-1010.

6. $\quad$ Clare, R. H.; Bardelle, C.; Harper, P.; Hong, W. D.; Börjesson, U.; Johnston, K. L.; Collier, M.; Myhill, L.; Cassidy, A.; Plant, D.; Plant, H.; Clark, R.; Cook, D. A. N.; Steven, A.; Archer, J.; McGillan, P.; Charoensutthivarakul, S.; Bibby, J.; Sharma, R.; Nixon, G. L.; Slatko, B. E.; Cantin, L.; Wu, B.; Turner, J.; Ford, L.; Rich, K.; Wigglesworth, M.; Berry, N. G.; O'Neill, P. M.; Taylor, M. J.; Ward, S. A., Industrial scale high-throughput screening delivers multiple fast acting macrofilaricides. Nat Commun 2019, 10 (1), 11-11.

7. Wölcke, J.; Ullmann, D., Miniaturized HTS technologies - uHTS. Drug Discovery Today 2001, 6 (12), 637-646.

8. Burbaum, J. J.; Sigal, N. H., New technologies for high-throughput screening. Current Opinion in Chemical Biology 1997, 1 (1), 72-78.

9. Major, J., Challenges and Opportunities in High Throughput Screening: Implications for New Technologies. Journal of Biomolecular Screening 1998, 3 (1), 13-17. 
10. Keserü, G. M.; Makara, G. M., The influence of lead discovery strategies on the properties of drug candidates. Nature Reviews Drug Discovery 2009, 8 (3), 203-212.

11. Zheng, B.; Roach, L. S.; Ismagilov, R. F., Screening of Protein Crystallization Conditions on a Microfluidic Chip Using Nanoliter-Size Droplets. Journal of the American Chemical Society 2003, 125 (37), 11170-11171.

12. Tarn, M. D.; Sikora, S. N. F.; Porter, G. C. E.; O’Sullivan, D.; Adams, M.; Whale, T. F.; Harrison, A. D.; Vergara-Temprado, J.; Wilson, T. W.; Shim, J.-u.; Murray, B. J., The study of atmospheric ice-nucleating particles via microfluidically generated droplets. Microfluidics and Nanofluidics 2018, 22 (5), 52.

13. Sjostrom, S. L.; Bai, Y.; Huang, M.; Liu, Z.; Nielsen, J.; Joensson, H. N.; Andersson Svahn, H., High-throughput screening for industrial enzyme production hosts by droplet microfluidics. Lab on a Chip 2014, 14 (4), 806-813.

14. Stan, C. A.; Schneider, G. F.; Shevkoplyas, S. S.; Hashimoto, M.; Ibanescu, M.; Wiley, B. J.; Whitesides, G. M., A microfluidic apparatus for the study of ice nucleation in supercooled water drops. Lab on a Chip 2009, 9 (16), 2293-2305.

15. Tjhung, K. F.; Burnham, S.; Anany, H.; Griffiths, M. W.; Derda, R., Rapid Enumeration of Phage in Monodisperse Emulsions. Analytical Chemistry 2014, 86 (12), 56425648.

16. Vogelstein, B.; Kinzler, K. W., Digital PCR. Proceedings of the National Academy of Sciences 1999, 96 (16), 9236.

17. Kang, H. M.; Subramaniam, M.; Targ, S.; Nguyen, M.; Maliskova, L.; McCarthy, E.; Wan, E.; Wong, S.; Byrnes, L.; Lanata, C. M.; Gate, R. E.; Mostafavi, S.; Marson, A.; Zaitlen, N.; Criswell, L. A.; Ye, C. J., Multiplexed droplet single-cell RNA-sequencing using natural genetic variation. Nature Biotechnology 2018, 36 (1), 89-94.

18. Macosko, Evan Z.; Basu, A.; Satija, R.; Nemesh, J.; Shekhar, K.; Goldman, M.; Tirosh, I.; Bialas, Allison R.; Kamitaki, N.; Martersteck, Emily M.; Trombetta, John J.; Weitz, David A.; Sanes, Joshua R.; Shalek, Alex K.; Regev, A.; McCarroll, Steven A., Highly Parallel Genome-wide Expression Profiling of Individual Cells Using Nanoliter Droplets. Cell 2015, 161 (5), 1202-1214.

19. Kawaguchi, M., Silicone oil emulsions stabilized by polymers and solid particles. Advances in Colloid and Interface Science 2016, 233, 186-199.

20. Bigg, E. K., The Supercooling of Water. Proceedings of the Physical Society. Section B 1953, 66 (8), 688-694.

21. Kay, J. E.; Tsemekhman, V.; Larson, B.; Baker, M.; Swanson, B., Comment on evidence for surface-initiated homogeneous nucleation. Atmos. Chem. Phys. 2003, 3 (5), 14391443.

22. Niedermeier, D.; Shaw, R. A.; Hartmann, S.; Wex, H.; Clauss, T.; Voigtländer, J.;

Stratmann, F., Heterogeneous ice nucleation: exploring the transition from stochastic to singular freezing behavior. Atmos. Chem. Phys. 2011, 11 (16), 8767-8775. 
23. Murray, B. J.; Broadley, S. L.; Wilson, T. W.; Atkinson, J. D.; Wills, R. H., Heterogeneous freezing of water droplets containing kaolinite particles. Atmos. Chem. Phys. 2011, 11 (9), 4191-4207. 
A

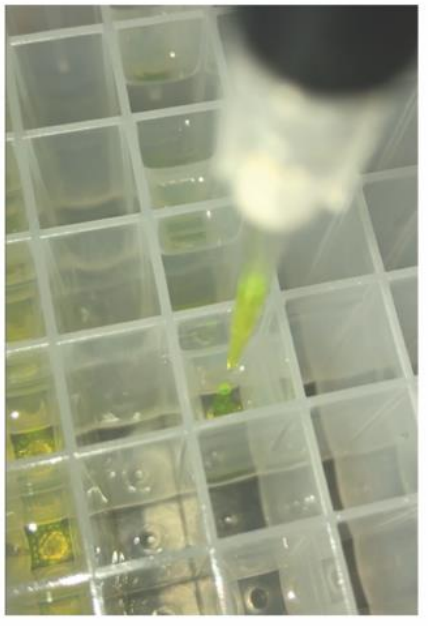

B
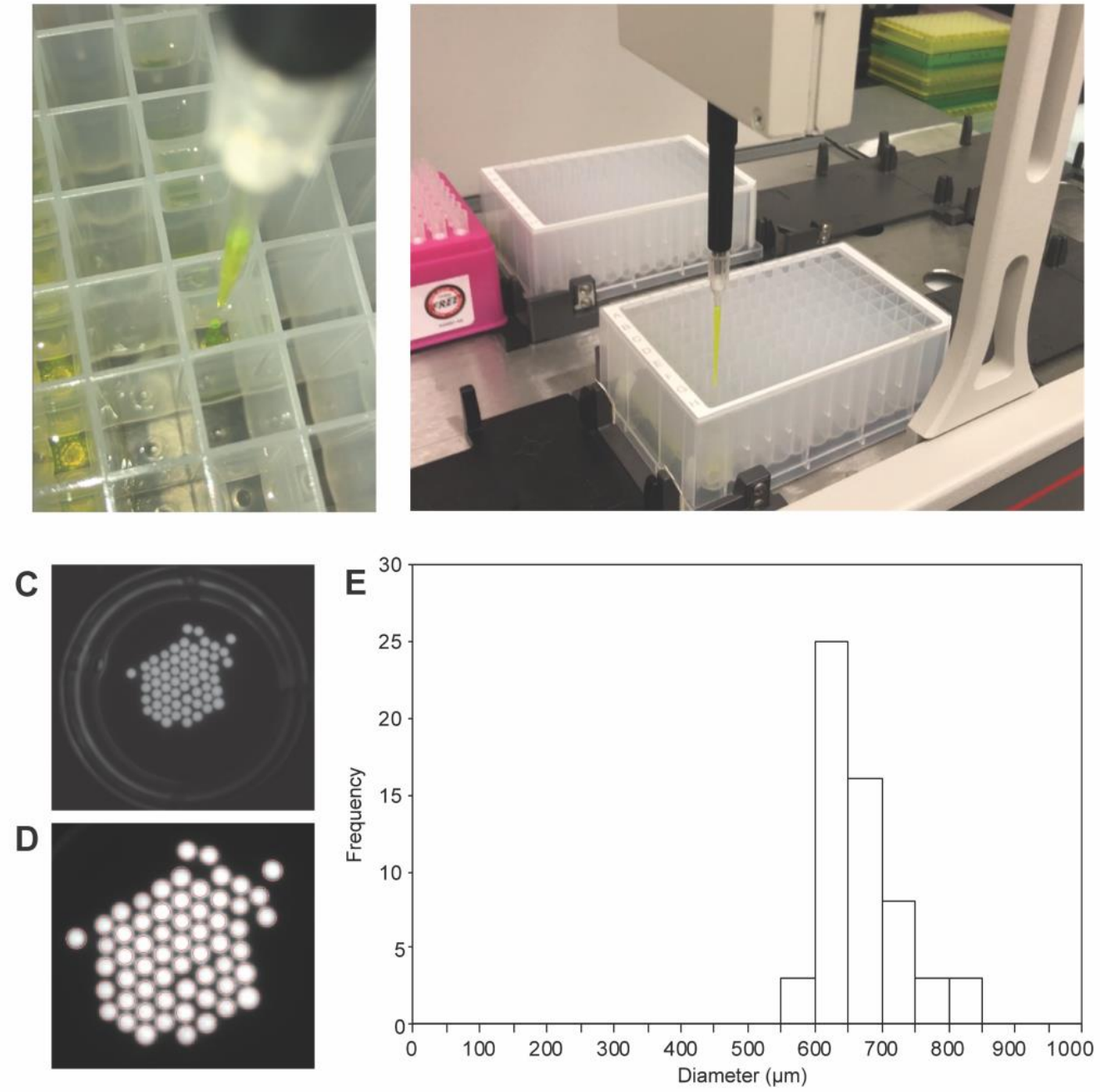

Figure 1. Automated droplets generation system using Biomek 3000. (A) Digital imaging of the automatic droplet generation. $1 \mu \mathrm{L}$ droplets were dispensed into the deep wells by the repeat pipetting. (B) Overview of the droplets generation machine. (C) Fluorescent imaging of the droplets. (D) Droplet detection system by Matlab. (E) Histogram of estimated droplets size. The droplets' polydispersity was $9.3 \%$. 

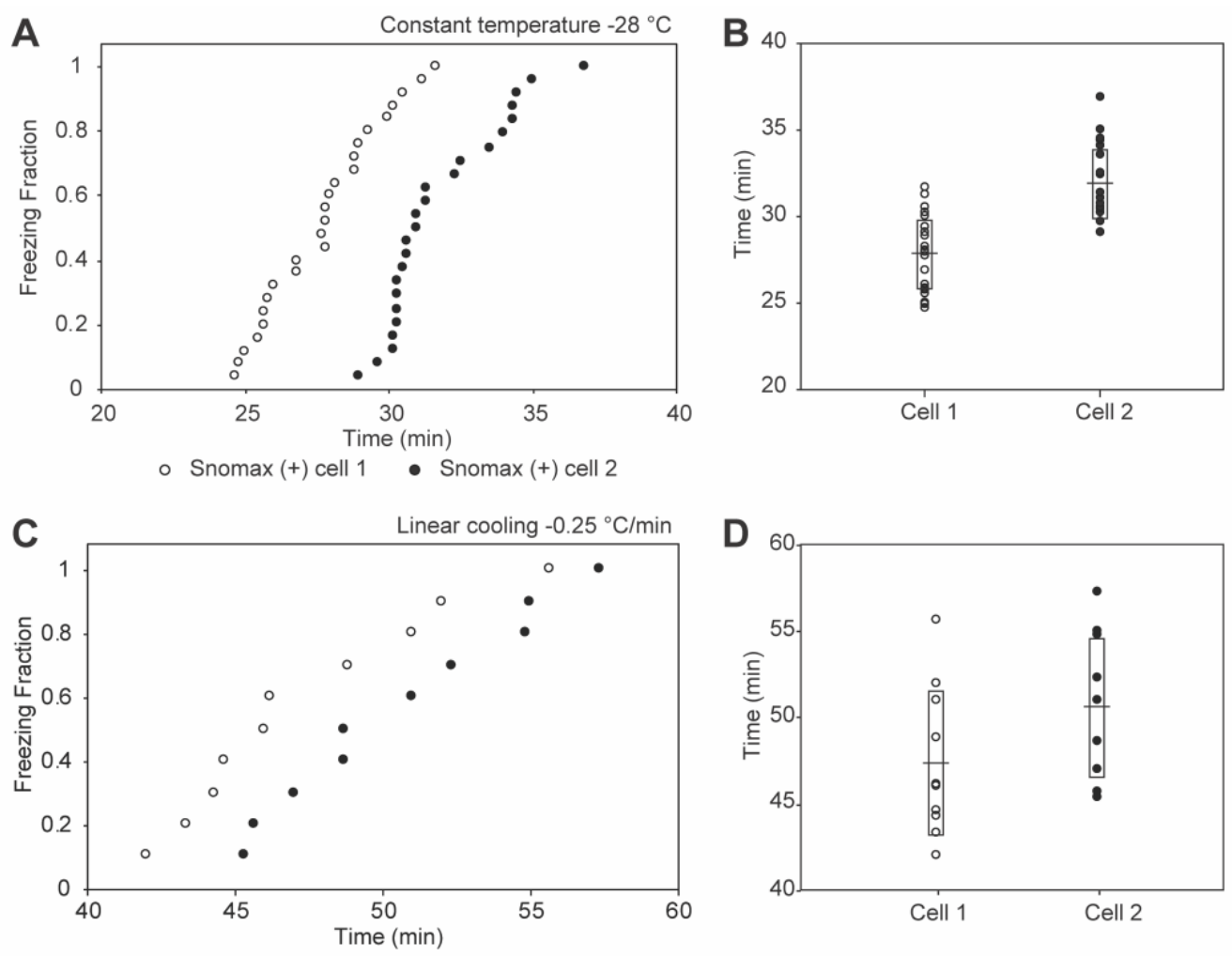

- Snomax $(+)$ cell 1 - Snomax $(+)$ cell 2

Figure 2. Thermal gradient in Biomek 3000 deep well system. (A) and (B) show the freezing temperature dispersion on constant temperature cooling at $-28{ }^{\circ} \mathrm{C}$ in different position of the deep well, and (C) and (D) show the linear cooling at $-0.25^{\circ} \mathrm{C} / \mathrm{min}$ in different position of the deep well. Freezing temperatures of $1 \mathrm{mg} / \mathrm{mL}$ Snomax droplets were measured over time. 
Const 1 vs Const 2


Figure 3. Comparison of droplet freezing temperature dispersions by Kolmogorov-Smirnov test. These results show the comparison of the cells in different position. Commodity freezer system with constant temperature $\left(\right.$ at $-27^{\circ} \mathrm{C}$ ) was used. Both used $1 \mathrm{mg} / \mathrm{mL}$ Snomax droplets. 

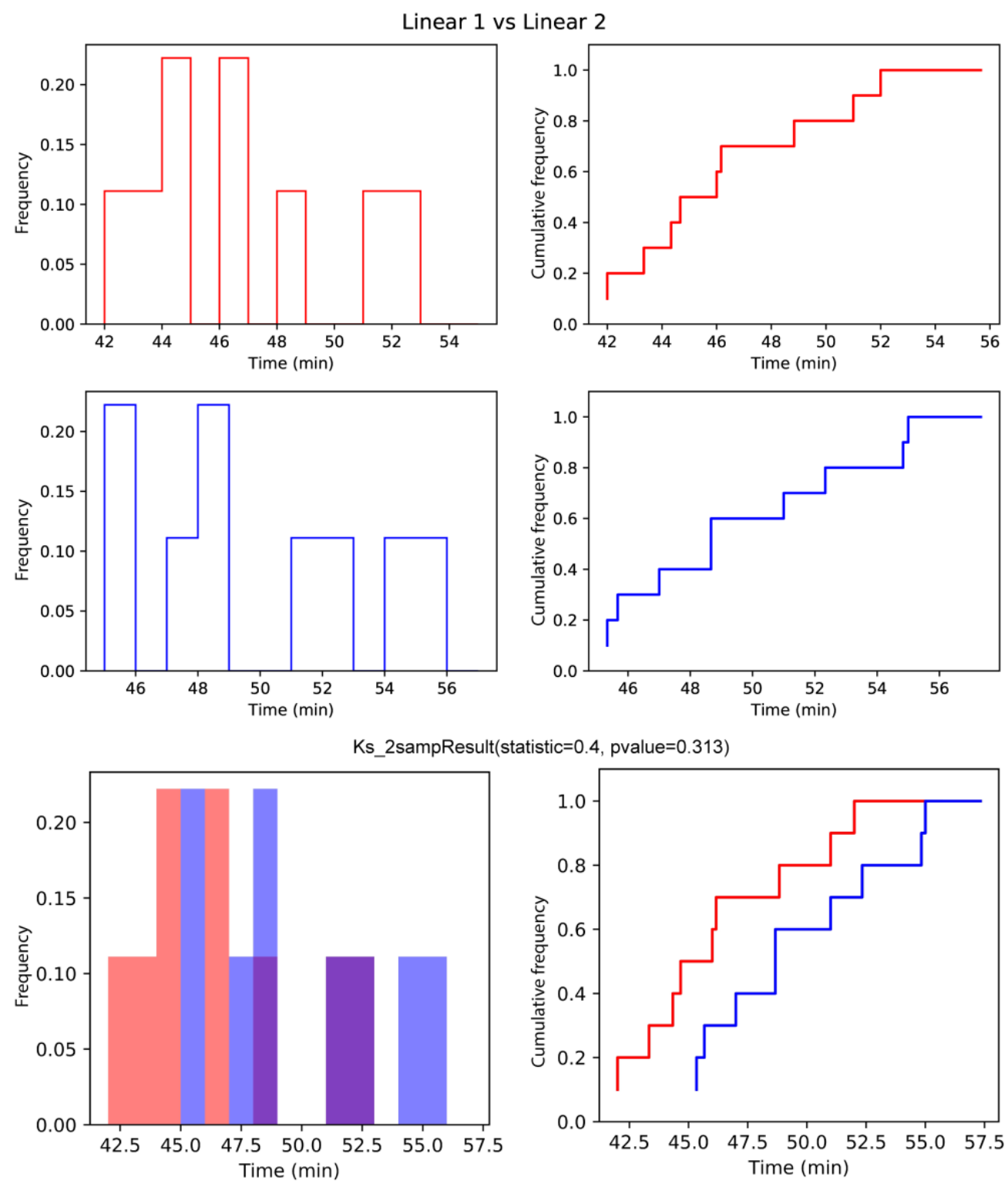

Figure 3. (Cont.) Comparison of droplet freezing temperature dispersions by KolmogorovSmirnov test. These results show the comparison of the cells in different position. Commodity freezer system with linear cooling $\left(-0.25^{\circ} \mathrm{C} / \mathrm{min}\right)$ was used. Both used $1 \mathrm{mg} / \mathrm{mL}$ Snomax droplets. 

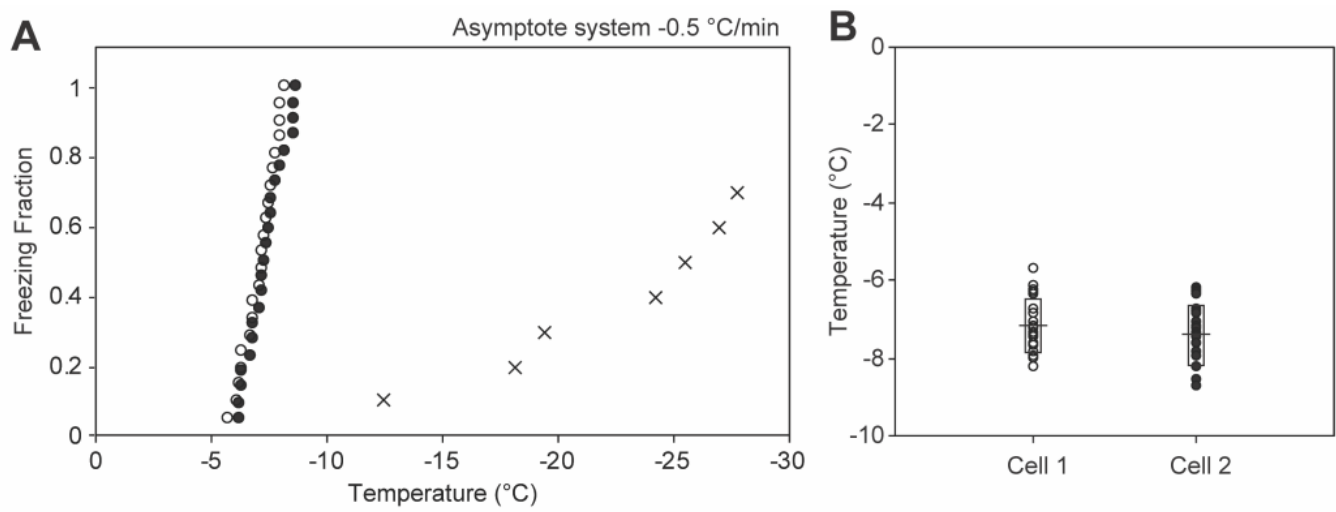

○ Snomax $(+)$ cell 1 - Snomax $(+)$ cell $2 \times \operatorname{Snomax}(-)$
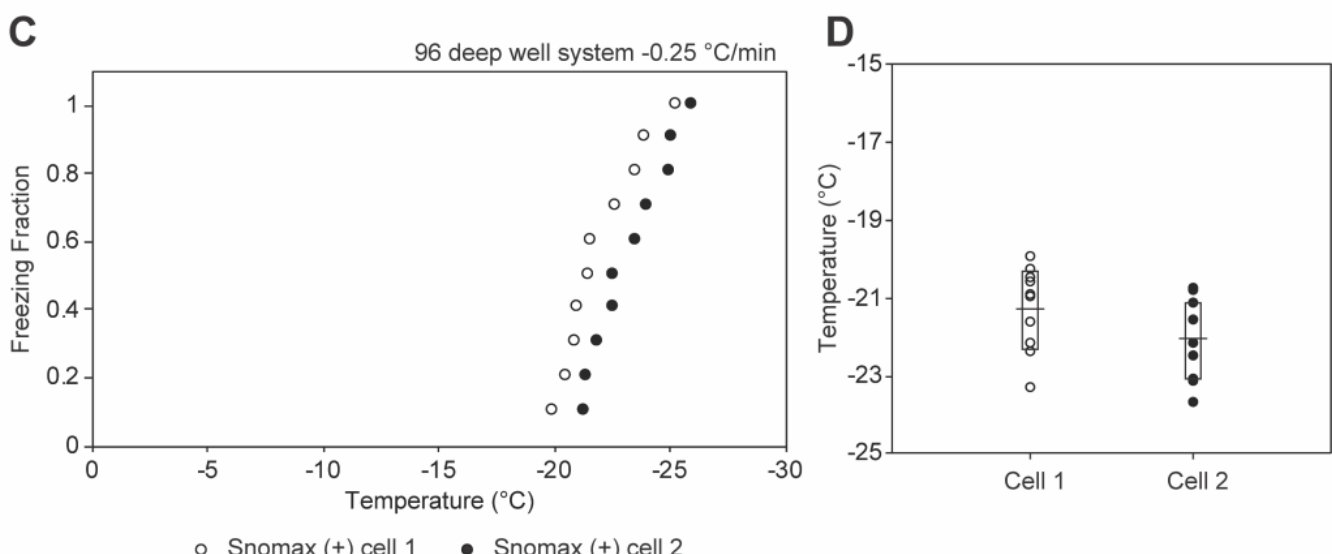

- Snomax $(+)$ cell 1 - Snomax $(+)$ cell 2

Figure 4. Thermal gradient in Asymptote system. (A-B) Asymptote constant-rate freezer with cooling rate at $-0.5{ }^{\circ} \mathrm{C} / \mathrm{min}$ in different position and (C-D) 96 deep well system with cooling rate at $-0.25^{\circ} \mathrm{C} / \mathrm{min}$ in different position as a reference. Freezing temperatures of two groups of droplets, containing either $1 \mathrm{mg} / \mathrm{mL}$ Snomax in water or water alone, were measured over time. 
Asy 1 vs Asy 2
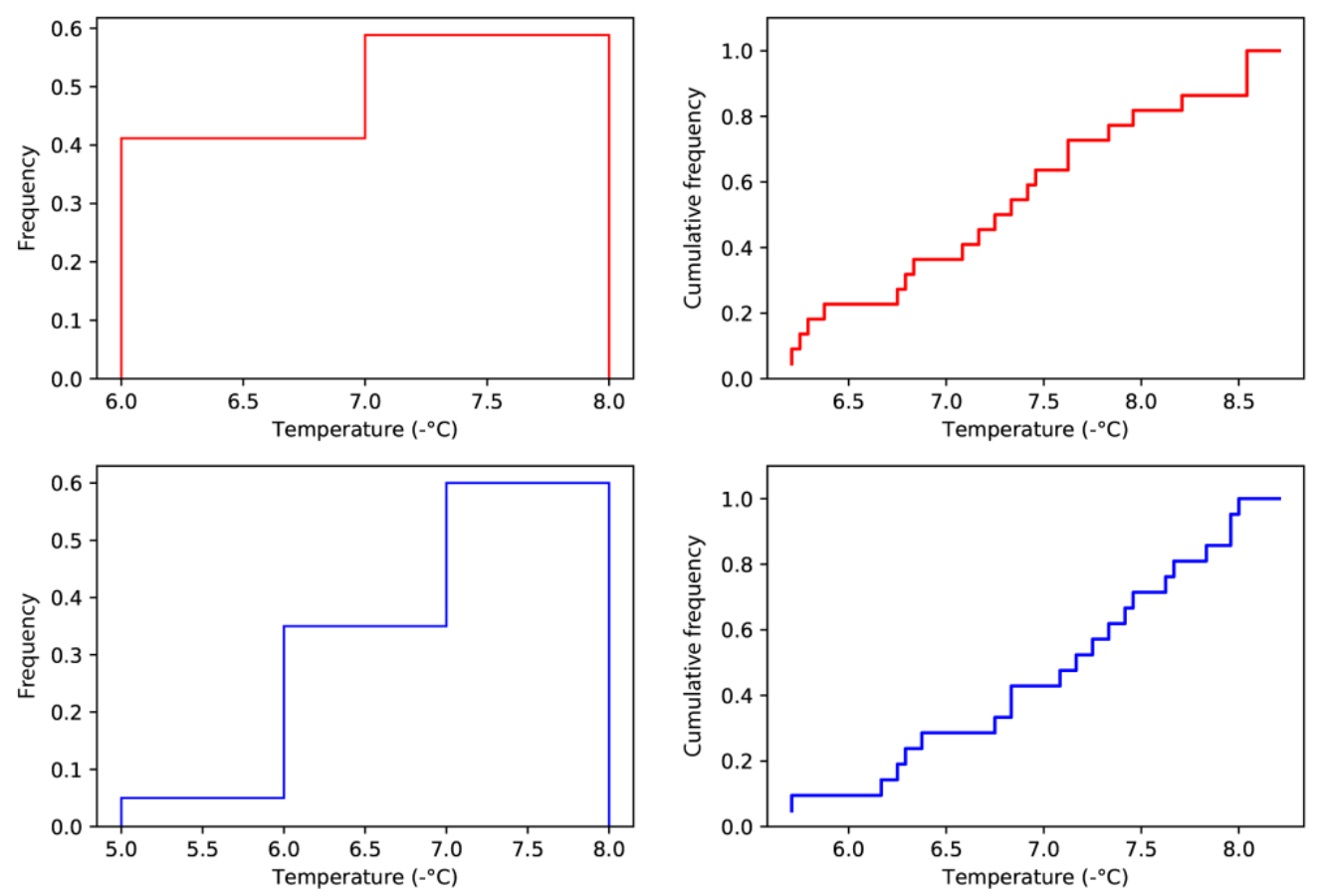

Ks_2sampResult(statistic $=0.182$, pvalue $=0.831$ )


Figure 5. Comparison of droplet freezing temperature dispersions by Kolmogorov-Smirnov test. These results show the comparison of the cells in different position cooled with Asymptote freezer (cooling rate $-0.5^{\circ} \mathrm{C} / \mathrm{min}$ ). Both used $1 \mathrm{mg} / \mathrm{mL}$ Snomax droplets. 
DW 1 vs DW 2
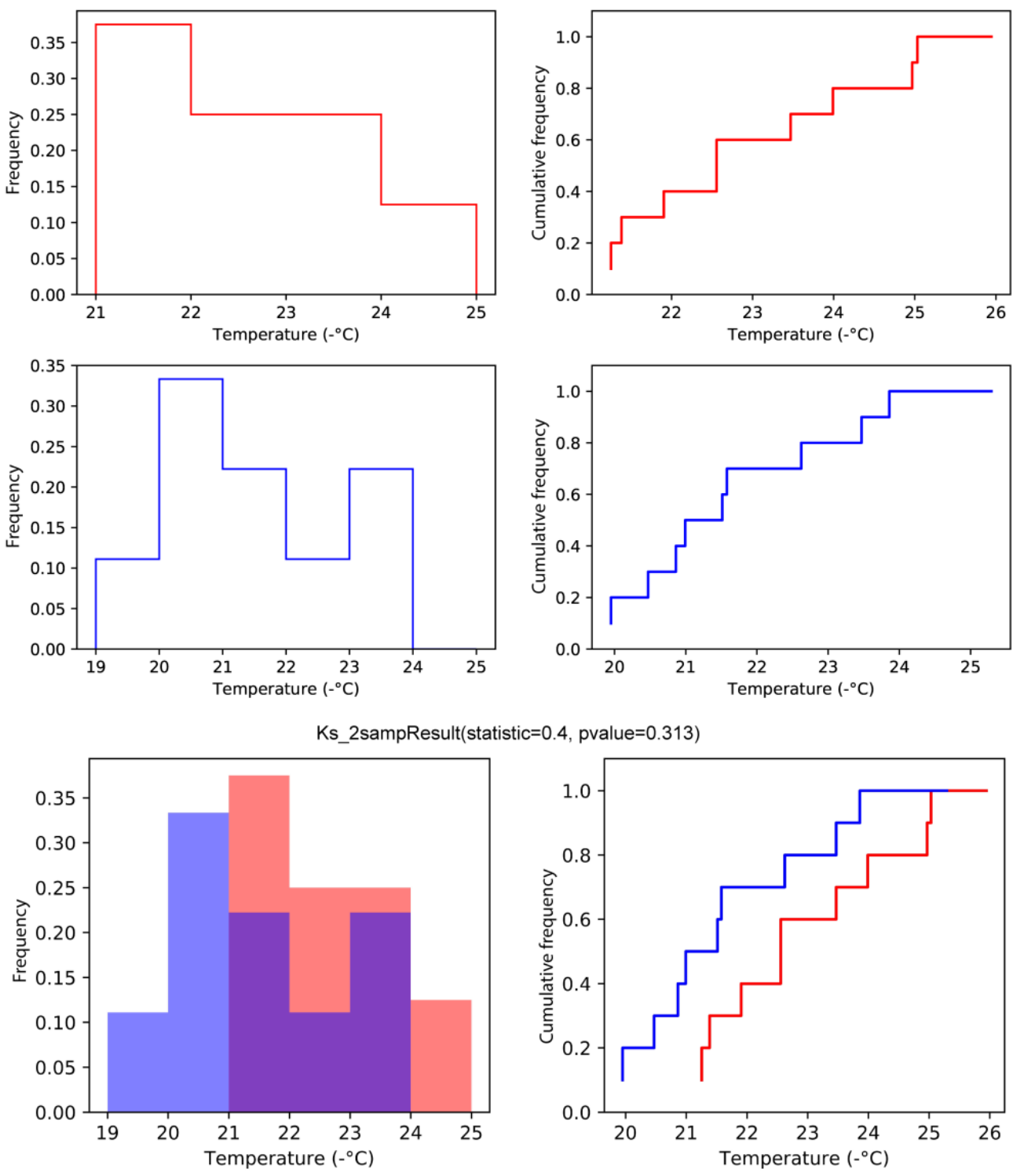

Figure 5. (Cont.) Comparison of droplet freezing temperature dispersions by KolmogorovSmirnov test. These results show the comparison of the deep well cells in different position cooled with commodity freezer system (linear cooling at $-0.25^{\circ} \mathrm{C} / \mathrm{min}$ ). Both used $1 \mathrm{mg} / \mathrm{mL}$ Snomax droplets. 

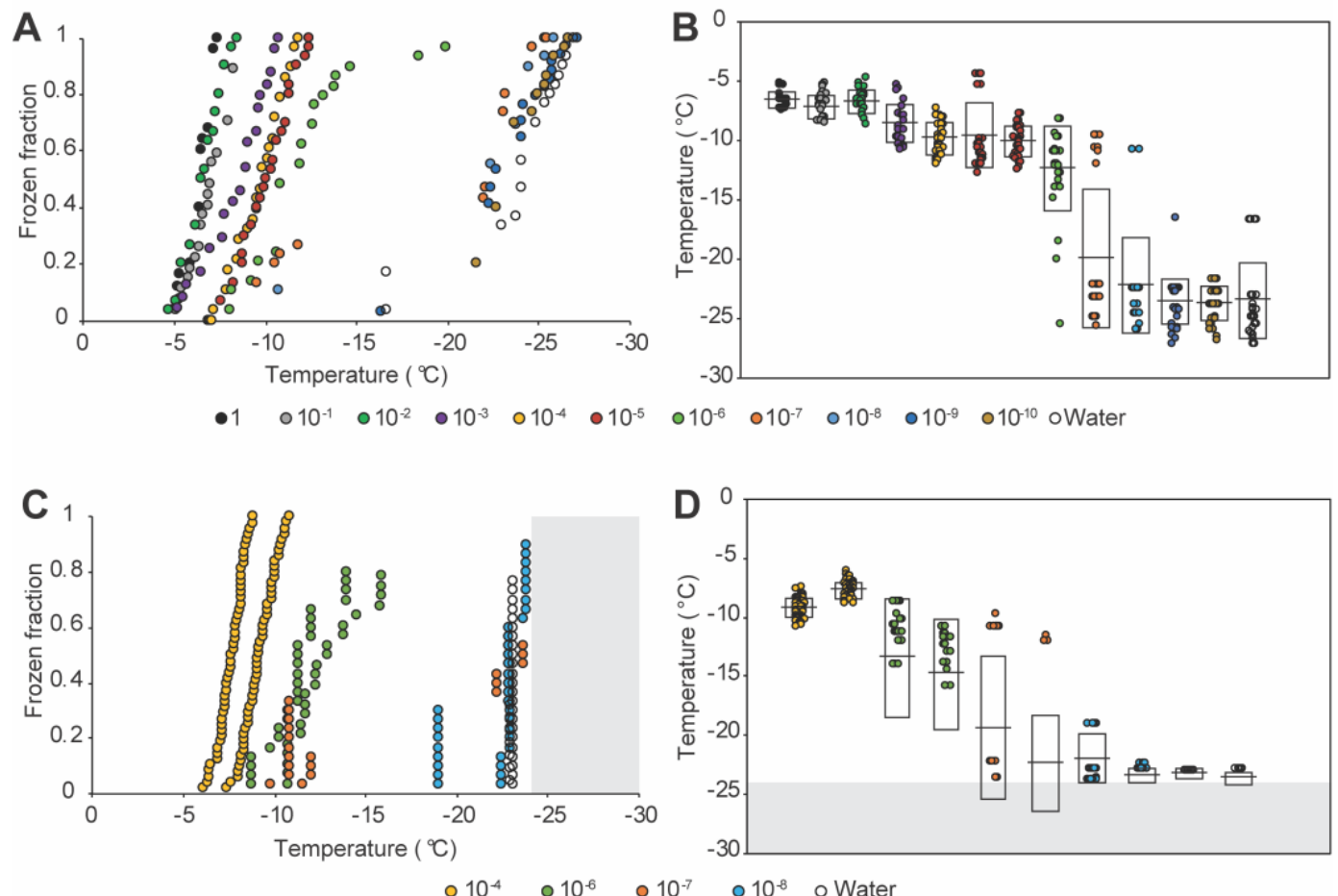

Figure 6. Droplet freezing temperatures of serial dilution of INA solution. Freeze-float selection system was cooled either (A, B) Asymptote freezing system or (C, D) generic freezer system. 